\section{Surgery Combined with LPRF in Denosumab Osteonecrosis of the Jaw: Case Report}

Gustavo Maluf1, Milena Correia de Pinho², Sandra Ribeiro de Barros da Cunha $^{3}$, Paulo Sérgio da Silva Santos ${ }^{4}$, Eduardo Rodrigues Fregnani
'Department of Dentistry, Bauru Dental School, USP - Universidade de São Paulo, Bauru, SP, Brazil ${ }^{2}$ Department of Oral Medicine, Hospital Sírio-Libanês, São Paulo, SP, Brazil ${ }^{3}$ Department of Dentistry, Dental School, USP - Universidade de São Paulo, São Paulo, SP, Brazil ${ }^{4}$ Department of Stomatology, Bauru Dental School, USP - Universidade de São Paulo, Bauru, SP, Brazil

Correspondence: Dr. Eduardo Rodrigues Fregnani, Rua Adma Jafet, 91, 01308-050 São Paulo, SP, Brasil. Tel: +55-11-3394-5369. e-mail: eduardofregnani@me.com
Key Words: osteonecrosis, jaws, denosumab, oncology.

\section{Introduction}

Denosumab is an antiresorptive drug used for the management of postmenopausal osteoporosis and metastatic bone disease. This antiresorptive agent is a human monoclonal antibody of nuclear factor kappa-B ligand (RANKL) that inhibits the development and activity of osteoclasts by blocking the binding of RANKL to RANK, decreasing bone resorption and increasing bone density (1-3).

This drug has a different mechanism of action from that of bisphosphonates. While bisphosphonates act targeting active mature osteoclasts, denosumab acts on osteoclast precursors via inhibition of RANKL, preventing their formation, differentiation and function as well as the associated bone resorption (3-5).

Recent literature has several publications, the majority of which investigating the effects of denosumab in comparison with those of bisphosphonates. In terms of adverse effects, osteonecrosis of the jaw (ONJ) is one of the most relevant, with an incidence ranging from 0.9 to $5 \%$ and the overall incidence of denosumab-related osteonecrosis of the jaw (DRONJ) is similar to that of bisphosphonate-related osteonecrosis of the jaw (DRONJ) in this population, ranging between 1-2\% (6).

Medication-related osteonecrosis of the jaw (MRONJ) can be defined as the exposure of necrotic bone in the maxillofacial region for more than 8 weeks in patients with history of using antiresorptive or antiangiogenic agents and no history of radiotherapy to the jaws (5).
MRONJ was first reported in 2003, but its pathophysiology has not yet been fully elucidated $(5,7,8)$. Some proposed hypotheses include inhibition of osteoclastic bone resorption and remodeling, inflammation and infection, inhibition of angiogenesis, soft tissues toxicity and immune dysfunction (5,9-11).

It has been noted that the risk of MRONJ in cancer patients exposed to denosumab is comparable to the risk of MRONJ in patients exposed to zolendronate, (70 up to 90 cases per 10,000 patients) $(5,10,12,13)$.

Regarding treatments, since its patophysiology cannot be explained with only one hypothesis and it can be considered as a multifactorial treatment, it is unlikely to exist only one successful treatment.

The approach to MRONJ should be multidisciplinary and include an appropriated dental evaluation before initiating the antiresorptive or antiangiogenic to reduce local risk factors $(5,14-18)$. Whenever MRONJ cannot be avoided, local treatment consists in eliminating the pain and control local infection. Literature divergences in treatment modalities such as conservative therapies (long-term antibiotic treatment and local disinfectant rinses) or surgical removal of the necrotic tissue with sequestromy and resection, but there is no report of the use of leucocyte-rich and platelet-rich fibrin (LPRF) associated with bone resection with patients with denosumab-related osteonecrosis of the jaw $(5,19,20)$.

This paper describes two clinical cases in which the use of LPRF combined with bone resection did not 
result in complete tissue response in the treatment of MRONJ.

\section{Case Report}

\section{Case 1}

A 69-year-old male, under treatment for metastatic lung cancer to bone and liver, making continuous use of Denosumab for the past 8 months and also current chemotherapy treatment with Irinotecan, sought our service with the complaint of pain for the previous 4 months from an implant surgery done 2 years before in the region of mandibular left first and second premolars and first molar and reopening 2 months before the consultation. On clinical exam, it was noted an area of bone exposure around the mandibular left first molar implant with purulent drainage associated with pain. With the clinical and tomographic diagnosis of MRONJ Stage 2, according to Ruggiero et al. (5), antibiotic therapy was started with amoxicillin/ clavulanate and the medical team discontinued the use of Denosumab for 4 months before the proposed surgical procedure. Surgical treatment was performed, as described below, in the Surgical Procedure section. Clinical followup of 4 months showed the patient to be asymptomatic

\section{Case 2}

A 44-year-old woman under treatment for breast cancer with bone metastasis making use of Bevacizumab and Denosumab for the past 7 months, presented for evaluation after endodontic surgery in the region of the mandibular left first molar associated with an allogenic bone graft performed 4 months previously. On clinical examination, it was noted bone exposure and purulent draining in the region from the mandibular left first premolar to second molar associated with important pain for the previous 3 months. The patient also reported, the use of Decadron and Fulvestrant. With clinical and tomographic diagnosis of MRONJ Stage 2, according to Ruggiero et al. (5), antibiotic therapy with penicillin/clavulanate was started and the medical team discontinued the use of Bevacizumab and Denasumab for 30 days before the proposed surgical procedure. The surgical procedure was performed according to the protocol described below in the Surgical Procedure section. With clinical follow-up for 6 months, the patient was asymptomatic, however there was partial mucosal wound covering.

\section{Surgical Procedure}

Both patients were medicated with analgesics
(Paracetamol $750 \mathrm{mg}$ and Nimesulide $100 \mathrm{mg}$ ), antibiotics (Penicilin/Clavulanate $875 \mathrm{mg}$ ) in addition to oral hygiene with $0.12 \%$ chlorhexidine ( $10 \mathrm{~mL}$, twice a day), preoperatively. Cone beam computed tomography scanning was performed for assessment of the extent of the bone necrosis. The surgical protocol included resection of the necrotic tissues, removal of granulation tissue and osteotomy with a rotary instrument until the presence of bleeding bone and rounding of all the sharp bone margins. In Case 2, we performed removal of the implants with retrieval key, minimizing damage to the bone tissue. Afterwards saline solution was used to perform intensive irrigation in order to remove debris, foreign bodies and clots. After this, we proceeded with the application of LPRF and primary closure with Cytoplast and/or Nylon. For LPRF, 10 to $30 \mathrm{~mL}$ of blood was collected. The blood samples collected in a $10 \mathrm{~mL}$ tube without an anticoagulant agent were immediately centrifuged at $2700 \mathrm{rpm}$ for 12 min in an LPRF Process Centrifugal (Intra-Lock System). The bone fragments removed were sent for histopathological investigation, which confirmed the clinical hypothesis of DRONJ.

Figures 1 and 2 illustrate the operative procedures of Cases 1 and 2, respectively.

\section{Discussion}

The search for medications with the same therapeutic effectiveness as the BP (bisphosphonates) but with fewer side effects has resulted in the discovery of Denosumab, which is a human monoclonal antibody to the receptor activator of nuclear factor kappa-B that inhibits the development and activity of osteoclasts by blocking the binding of RANKL to RANK, decreasing bone resorption, and increasing bone density $(1,2)$.

The adverse effects of Denosumab and bisphosphonates have been investigated and MRONJ, a major adverse condition commonly associated with both drugs, has a similar incidence in patients using Denosumab and bisphosphonates (6)

Development of MRONJ can occur spontaneously or may be facilitated by tooth extraction, periapical surgery, periodontal surgery involving osseous tissue, local trauma, dental procedures, poor oral hygiene and actinomyces infections (5). Both cases reported here occurred after surgical procedures (reopening of the implants in Case 1 and periapical surgery in Case 2). Literature shows that the maxilla seems to be less affected than the mandible, which is also confirmed by both of our cases, which affected the posterior mandible (5).

The first objective in the treatment of MRONJ is to alleviate pain, reduce infection and stabilize the disease progression with the closure of bone exposure. Most 
published cases, which can be associated with a RANKligand inhibitor administration, have predominantly been managed conservatively, using long-term antibiotic treatment and local disinfectant rinses. (5) These treatment regimens have been reported to be unsuccessful in at least $60 \%$ of the cases. The more experience with MRONJ is gathered, the more surgical therapy is recommended as success rates are higher, disease progression can be halted, and the diagnosis of osteonecrosis can be proven histologically, while other causes for exposed bone can be excluded.

There is a shortage of case report focusing on the treatment of DRONJ, therefore there is no standard treatment for this pathology. In the reviewed literature, the most used treatment is the same protocol for BRONJ, which consists of surgery in order to remove the necrotic bone combined with a long-term antibiotic therapy $(22,23)$.

Unlike bisphosphonates, the antiresorptive effects of Denosumab decrease with time. It is recommended to discontinue the therapy preferably for at least 3 months, if possible for 6 months. Despite the lack of studies supporting or refuting this drug withdrawing, this is a crucial difference from the recommendations for BRONJ treatment (6). Denosumab was discontinued for 4 months in Case 1 and 30 days in Case 2.

It appears that the use of LPRF has never been described before as an adjuvant treatment for DRONJ, although some have been reported the use of bone anabolic agent such as TPD (teriparatide) associated with surgery and antibiotics and fluorescence guided bone ressection $(21,24)$.

Dell Fabbro et al. (25), in a systematic review, suggested that the use of platelet concentrations as an adjunct to
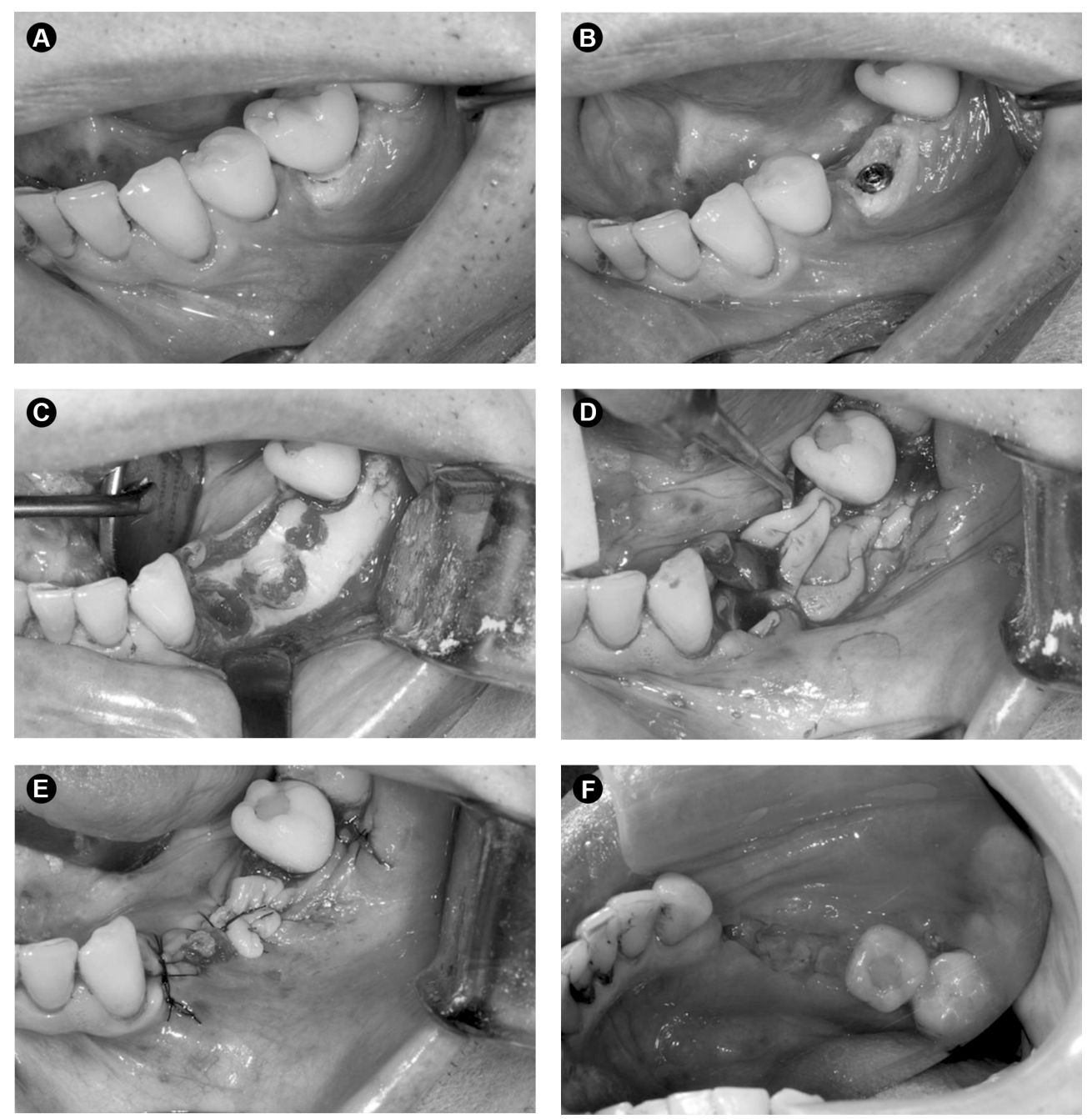

Figure 1. A: Area of bone exposure around the mandibular left first molar implant with purulent drainage. B: Clinical aspect after removal of the implant crown. C: Trans-surgical aspect, after resection of necrotic tissues, removal of granulation tissue, removal of implants, osteotomy with rotary instrument until the presence of bleeding bone, and rounding of all the sharp bone margins. D: Application of leucocyte-rich and platelet-rich fibrin (LPRF) in the surgical recess. E: Primary closure with Nylon sutures. F: Postoperative period of 4 months showing partial mucosal wound covering. 
the oral surgery procedure may have a beneficial effect for the post-surgical prevention, occurrence or recurrence of MRONJ in patients under treatment with bisphosphonates.

In platelet rich plasma (PRP) with its pro-coagulant effects, the platelets form a rich source of important growth factors, such as those derived from platelets (PDGF), the transformation growth factor - $b$ (TGF- b) 1 and 2, and vascular endothelial growth factor (VEGF), all of these are involved in the angiogenic cascade that helps the healing of hard and soft tissues, which improve the healing of hard and soft tissues (26).

Some authors have used PRP in MRONJ as adjuvant therapy to improve bone healing, lower need for analgesics and the resolution of lesions. Mozzati et al. (27) obtained positive results in 32 patients, which were treated with intravenous BP for oncological pathologies. Soydan and
Uckan (28) used two layers of PRF membrane after the surgical removal of MRONJ and in a follow-up of 4 months, there was complete close of the bone exposure.

LPRF has superior characteristics in the healing process than the PRP by stimulating the repair of soft tissues and acting as a membrane barrier between alveolar bone and the oral cavity. In addition, LPRF leukocytes pursue an immune regulation, which may be important in preserving tissue infection in the immediate postoperative period (13). The mature and dense LPRF fibrin network allows the storage of cells such as platelets, so that there is slow release of growth factors (29). Kim et al. (30) evaluated 34 patients in which LPRF was combined with surgical resection. The evaluations were made after 4 months, with 26 (77\%) presenting complete resolution, $6(18 \%)$ having delay in resolution, and $2(6 \%)$ showing no resolution.
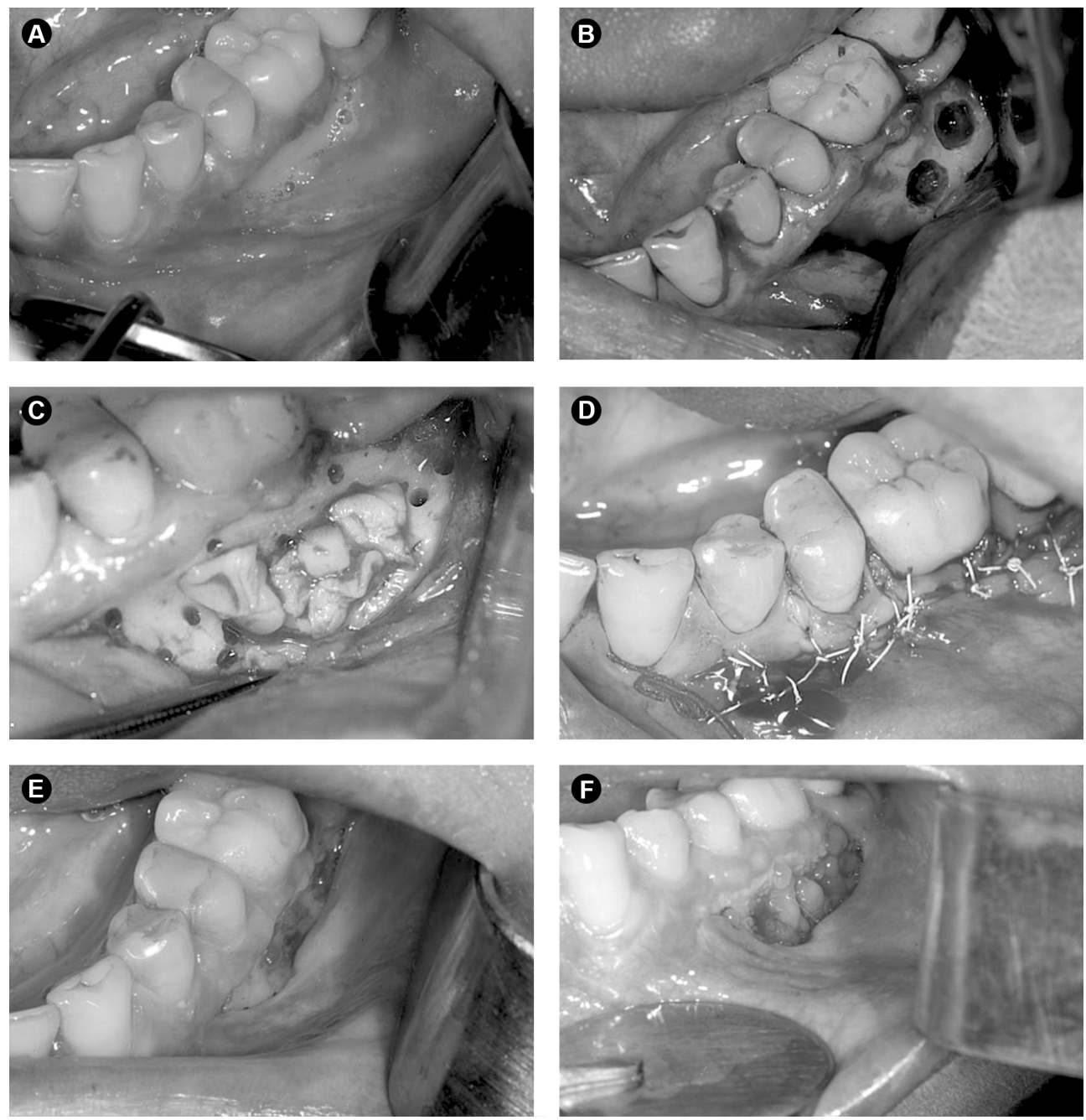

Figure 2. A: Bone exposure and purulent drainage in the region from the mandibular left first premolar to the second molar associated with previous endodontic surgery. B: Trans-surgical aspect after flap, showing sites of previous endodontic surgery. C: Application of leucocyte-rich and platelet-rich fibrin (LPRF) in surgical recess, after resection of necrotic tissues, removal of granulation tissue, osteotomy with rotary instrument until the presence of bleeding bone and rounding of all the sharp bone margins. D: Suture of surgical recess with primary closure. E: Clinical aspect in post-operative period of 30 days, showing no closure of the surgical wound. F: Clinical aspect after a postoperative period of 4 months, with evident granulation tissue, however, exhibiting a partial mucosal wound covering. 
Studies associating LPRF with osteonecrosis of the jaw are scarce and in two of them $(28,30)$ the antiresorptive agents were bisphosphonates unlike our cases where they were used the Denosumab (Case 1) and Denosumab with Bevacizumab (Case 2). Despite the new AAOMS recommendations, success rates of conservative treatment regimens range from less than $20 \%$ to over $50 \%$, which is significantly lower than the success rate of over $85 \%$ reported for surgical therapy approaches. The qualitative definition of successful therapy may be a contributing factor to the discrepancy observed in the success rate of conservative treatments. By minimizing symptoms and infections an intermittent improvement may be achieved, but it should not be classed as successful therapy for the disease or elimination of the osteonecrosis. This notion might explain why many investigators correctly conclude that MRONJ is a relapsing and remitting diagnosis. Indeed, the conservative management is typically related to the palliation of osteonecrosis, whereas surgical resection most commonly translates into the cure of a patient's disease (31).

Regarding the cases presented herein using PRP and LPRF, in the follow-up of 4 and 6 months, both patients had not presented complete healing of the surgical wound which has a different outcome that literature reports with BRONJ cases $(27,28)$. This may be justified by the observation of Zebaze et al. (32), who reported that denosumab reduced remodeling more rapidly and more completely than alendronate.

Literature points it out that patients treated with anti-tumor therapies targeting VEGF-A, such as Bevacizumab, have also been linked to MRONJ as a side effect. Following the evidence that VEGF is essential for osteogenic differentiation and bone formation, it has been hypothesized that the anti-angiogenic properties of Bevacizumab could compromise microvessel integrity in the jaw and lead to subclinical compromise of the bone. Similarly, impaired angiogenesis-dependent wound healing has been hypothesized to explain how Bevacizumab could cause oral mucosal breakdown and expose necrotic jawbone (5). In Case 2, the patient made concomitant use of Denosumab and Bevacizumab, a fact that could possibly have delayed an adequate repair when compared with previous literature follow-ups.

These case reports show that in addition to the importance of the dental surgeon being aware of all the treatments available for the management of osteonecrosis, the etiology of the disease may be a fundamental factor in cases of failure, even in surgical approaches using advanced techniques, and that alternative and new protocols must be investigate. The association of surgery with the use of growth factors has no side effects. Rather, this method incorporates the use of platelet concentrate rich in leukocytes and fibrin (LPRF) is characterized by no addition of chemicals, which allows the natural coagulation process and differs from the characteristics of PRP. Unlike PRP, LPRF does not dissolve quickly after use, preserving the fibrin matrix architecture and becoming a more favorable environment for the release of growth factors (29). The combination of surgery, PRP and LPRF could be a promising treatment for the cases of DRONJ.

Based on the literature and the findings of the reported cases, we can conclude that the use of LPRF can be considered in the treatment of patients with osteonecrosis of the jaw related to Denosumab.

\section{Resumo}

Este artigo descreve dois casos onde a ressecção óssea associada à fibrina rica em plaquetas e leucócitos (LPRF) não resultou em resposta completa no tratamento da osteonecrose dos maxilares relacionados à medicações (MRONJ). Como observado nos casos aqui relatados, MRONJ foi recentemente descrito na literatura em pacientes que recebem a administração subcutânea de inibidores-RANK, como Denosumab ou drogas anti-angiogenicas, como Bevacizumab. Estudos recentes com resultados promissores indicam que mais pacientes serão tratados com estas terapias para evitar complicações esqueléticas devido às metástases ósseas. Portanto, este cenário pode tornar-se um desafio clínico comparável às osteonecroses dos maxilares relacionados aos bisfosfonatos na área de Cirurgia Bucomaxilofacial. Até o momento, nenhuma técnica cirúrgica foi descrita com eficiência para superar as lesões da mucosa com exposição óssea e que não cicatrizam devido a terapia com inibidores de RANK. Com base na literatura e nos achados dos casos reportados, podemos concluir que o uso do LPRF pode ser considerado no tratamento de pacientes com osteonecrose dos maxilares relacionados ao Denosumab.

\section{References}

1. Malan J, Ettinger $K$, Naumann E, Beirne OR. The relationship of denosumab pharmacology and osteonecrosis of the jaws. Oral Surg Oral Med Oral Pathol Oral Radiol 2012 114:671-676.

2. Hoefert $S$, Hoefert CS, Albert M, Munz A, Grimm M, Northoff $H_{\text {, }}$ et al.. Zoledronate but not denosumab suppresses macrophagic differentiation of THP-1 cells. An aetiologic model of bisphosphonaterelated osteonecrosis of the jaw (BRONJ). Clin Oral Investig 2015;19:1307-1318.

3. Yamashita J, McCauley LK. Antiresorptives and osteonecrosis of the jaw. J Evid Based Dent Pract 2012;12:233-247.

4. Oliveira CC, Brizeno LA, Sousa FB, Mota MR, Alves AP. Osteonecrosis of the jaw induced by receptor activator of nuclear factor-kappa $B$ ligand (Denosumab). Med Oral Patol Oral Cir Bucal 2016 [Epub ahead of print. doi:10.4317/medoral.21044]

5. Ruggiero SL, Dodson TB, Fantasia J, et al. American Association of Oral and Maxillofacial Surgeons. American Association of Oral and Maxillofacial Surgeons position paper on medication-related osteonecrosis of the jaw - 2014 update. J Oral Maxillofac Surg 2014 72:1938-1956

6. Otto, S, Pautke, C. Treatment of medication-related osteonecrosis of the jaw. In (ed.), Medication Related Osteonecrosis of the Jaws: Bisphosphonates, Denosumab and New Agents. Springer- Verlog Berlin Heidelberg 79-92, 2015.

7. Marx RE. Pamidronate (Aredia) and zoledronate (Zometa) induced avascular necrosis of the jaws: A growing epidemic. J Oral Maxillofac Surg 2003;61:1115-1117.

8. Ruggiero SL, Mehrotra B, Rosenberg TJ, Engroff SL. Osteonecrosis of the jaws associated with the use of bisphosphonates: A review of 63 cases. J Oral Maxillofac Surg 2004 62:527-534.

9. Reid IR, Bolland MJ, Grey AB. Is bisphosphonate-associated 
osteonecrosis of the jaw caused by soft tissue toxicity? Bone 2007:41:318-320.

10. Bamias A, Kastritis E, Bamia C, Moulopoulos LA, Melakopoulos I, Bozas $G$, et al.. Osteonecrosis of the jaw in cancer after treatment with bisphosphonates: Incidence and risk factors. J Clin Oncol, 2005;23:8580-8587.

11. Wood J, Bonjean K, Ruetz S, Bellahcène A, Devy L, Foidart JM, et al.. Novel antiangiogenic effects of the bisphosphonate compound zoledronic acid. J Pharmacol Exp Ther 2002;302:1055-1061.

12. Fizazi $K$, Carducci $M$, Smith $M$, Damião $R$, Brown J, Karsh $L$, et al.. Denosumab versus zoledronic acid for treatment of bone metastases in men with castration-resistant prostate cancer: A randomised, doubleblind study. Lancet 2011;377:813-822.

13. Henry DH, Costa L, Goldwasser F, Hirsh V, Hungria V, Prausova J, et al.. Randomized, double- blind study of denosumab versus zoledronic acid in the treatment of bone metastases in patients with advanced cancer (excluding breast and prostate cancer) or multiple myeloma. J Clin Oncol 2011;29:1125-1132.

14. Dimopoulos MA, Kastritis E, Bamia C, Melakopoulos I, Gika D, Roussou $\mathrm{M}$, et al.. Reduction of osteonecrosis of the jaw (ONJ) after implementation of preventive measures in patients with multiple myeloma treated with zoledronic acid. Ann Oncol 2009;20:117-120.

15. Ripamonti $\mathrm{Cl}$, Maniezzo $\mathrm{M}$, Campa T, Fagnoni E, Brunelli C, Saibene $G$, et al.. Decreased occurrence of osteonecrosis of the jaw after implementation of dental preventive measures in solid tumor patients with bone metastases treated with bisphosphonates. The experience of the National Cancer Institute of Milan. Ann Oncol 2009;20:137-145.

16. Saad F, Brown JE, Van Poznak C, Ibrahim T, Stemmer SM, Stopeck AT, et al.. Incidence, risk factors, and outcomes of osteonecrosis of the jaw: Integrated analysis from three blinded active-controlled phase III trials in cancer patients with bone metastases. Ann Oncol 2012;23:13411347.

17. Bonacina R, Mariani U, Villa F, Villa A. Preventive strategies and clinical implications for bisphosphonate-related osteonecrosis of the jaw: $A$ review of 282 patients. J Can Dent Assoc 2011;77:b147

18. Vandone AM, Donadio M, Mozzati M, Ardine M, Polimeni MA, Beatrice $\mathrm{S}$, et al.. Impact of dental care in the prevention of bisphosphonateassociated osteonecrosis of the jaw: A single-center clinical experience. Ann Oncol 2012;23:193-200.

19. Ferlito S, Puzzo S, Palermo F, Verzi P. Treatment of bisphosphonaterelated osteonecrosis of the jaws: Presentation of a protocol and an observational longitudinal study of an Italian series of cases. Br J Oral Maxillofac Surg 2012;50:425-429.

20. Wutzl A, Biedermann E, Wanschitz F, Seemann R, Klug C, Baumann A, et al.. Treatment results of bisphosphonate-related osteonecrosis of the jaws. Head Neck 2008;30:1224-1230.

21. Otto $S$, Baumann $S$, Ehrenfeld $M$, Pautke C. Successful surgical management of osteonecrosis of the jaw due to RANK-ligand inhibitor treatment using fluorescence guided bone resection. J Craniomaxillofac Surg 2013;41:694-698.

22. Pichardo SE, Kuypers SC, van Merkesteyn JP. Denosumab osteonecrosis of the mandible: a new entity? A case report. J Craniomaxillofac Surg 2013;41:e65-e69.

23. Aghaloo TL, Felsenfeld AL, Tetradis S. Osteonecrosis of the jaw in a patient on Denosumab. J Oral Maxillofac Surg 2010;68:959-963.

24. Neuprez A, Coste S, Rompen E, Crielaard JM, Reginster JY. Osteonecrosis of the jaw in a male osteoporotic patient treated with denosumab. Osteoporos Int 2014;25:393-395.

25. Del Fabbro M, Gallesio G, Mozzati M. Autologous platelet concentrates for bisphosphonate-related osteonecrosis of the jaw treatment and prevention. A systematic review of the literature. Eur J Cancer 2015;51:62-74.

26. Nikolidakis $D$, Jansen JA: The biology of platelet-rich plasma and its application in oral surgery: literature review. Tissue Engineering: Part B 2015;14:249-258.

27. Mozzati M, Gallesio G, Arata V, Pol R, Scoletta M. Platelet-rich therapies in the treatment of intravenous bisphosphonate-related osteonecrosis of the jaw: A report of 32 cases. Oral Oncology 2012;48:469-474.

28. Soydan SS, Uckan S. Management of bisphosphonate-related osteonecrosis of the jaw with a platelet-rich fibrin membrane: technical report. J Oral Maxillofac Surg 2014;72:322-326.

29. Dohan DM, Choukroun J, Diss A, Dohan SL, Dohan AL, Mouhy J, et al.. Platelet-rich fibrin (PRF): A second-generation platelet concentrate. Part III: Leucocyte activation: A new feature for platelet concentrate? Oral Surg Oral Med Oral Pathol Oral Radiol Endod 2006;101:51-55.

30. Kim JW, Kim SJ, Kim MR. Leucocyte-rich and platelet-rich fibrin for the treatment of bisphosphonate-related osteonecrosis of the jaw: a prospective feasibility study. Br J Oral Maxillofac Surg 2014;52:854859.

31. Ristow 0,0 tto $\mathrm{S}$, Troeltzsch $\mathrm{M}$, Hohlweg-Majert B, Pautke C. Treatment perspectives for medication-related osteonecrosis of the jaw (MRONJ). J Craniomaxillofac Surg 2015;43:290-293.

32. Zebaze RM, Libanati $C$, Austin $M$, Ghasem-Zadeh $A$, Hanley DA, Zanchetta JR, et al.. Differing effects of denosumab and alendronate on cortical and trabecular bone. Bone 2014;59:173-179.

Received November 25, 2015 Accepted April 25, 2016 